\title{
Prevalencia de estrés, síndrome de desgaste profesional, ansiedad y depresión en médicos de un hospital universitario durante la pandemia de COVID-19
}

\author{
Prevalence of stress, burnout syndrome, anxiety and \\ depression among physicians of a teaching hospital during the \\ COVID-19 pandemic
}

Prof. Dr. Francisco J. Appiani ${ }^{a}$, Dr. Federico Rodríguez Cairoli ${ }^{a}$, Prof. Dr. Luis Sarotto (h) Dr. Claudio Yaryourc, Dra. María E. Basile y Dr. Juan M. Duarte ${ }^{a}$

\section{RESUMEN}

Introducción. Los trabajadores de la salud se encuentran sometidos a una gran tensión en el desarrollo de sus actividades, lo que genera alta frecuencia de estrés, desgaste laboral e impacto psicopatológico. La pandemia de COVID-19 podría provocar un incremento de estas entidades en los médicos. Elobjetivo fue describir la frecuencia de estrés, síndrome de desgaste profesional (burnout), ansiedad y depresión durante la pandemia, y analizar las asociaciones con distintas variables independientes.

Métodos. Estudio observacional, transversal, realizado dos meses después del inicio de la cuarentena en Argentina. Se encuestó a médicos de especialidades clínicas, quirúrgicas, solo de emergencias, y a aquellos sin contacto directo con pacientes, mediante un cuestionario sociodemográfico y tres inventarios autoadministrados: Health Professions Stress Inventory, Maslach Burnout Inventory y la Escala de ansiedad y depresión hospitalaria.

a. Unidad de Neuropsicofarmacología. Dirección de Docencia e Investigación.

b. Departamento de Cirugía.

c. División de Emergencias. Hospital de Clínicas José de San Martín, Universidad de Buenos Aires, Ciudad Autónoma de Buenos Aires, Argentina.

Correspondencia: Dra. María E. Basile: basileeuge@gmail.com

Financiamiento:

Ninguno.

Conflicto de intereses: Ninguno que declarar. http://dx.doi.org/10.5546/aap.2021.317

Texto completo en inglés:

http:/ / dx.doi.org/10.5546/ aap.2021.eng.317
Cómo citar: Appiani FJ, Rodríguez Cairoli F Sarotto (h) L, Yaryour C, et al. Prevalencia de estrés, síndrome de desgaste profesional, ansiedad y depresión en médicos de un hospital universitario durante la pandemia de COVID-19. Arch Argent Pediatr 2021;119(5):317-324.

\section{INTRODUCCIÓN}

La epidemia de la enfermedad por el nuevo coronavirus (COVID-19), causada por el coronavirus del 2019 (SARS-CoV-2), comenzó en China a fines de diciembre de ese año y se ha extendido en pocos meses por todo el mundo. ${ }^{1}$ Debido a su rápida difusión y las consecuencias clínicas, los recursos de salud se han visto sometidos a una gran presión.

El personal de salud, en particular los médicos, se ha visto especialmente afectado por esta pandemia. Por ejemplo, en Italia, el $20 \%$ de los trabajadores sanitarios que respondieron una encuesta estaban infectados y referían que algunos colegas habían muerto. ${ }^{2}$

Los médicos que se encuentran en la primera línea de atención se enfrentan a un estrés cada vez mayor debido a la gran carga de trabajo, al dolor de perder pacientes y colegas, junto con -en algunos casosdisponibilidad insuficiente de recursos (por ejemplo, el equipo de protección personal) o falta de capacitación en protocolos de protección. Esto está intrínsecamente relacionado con el miedo a infectarse, que puede ser un camino directo hacia el agotamiento psicofísico y los trastornos de salud mental. ${ }^{3}$ Pero el problema no se limita 
solo a quienes enfrentan la pandemia en la primera línea. Algunos médicos se ven expuestos a la suspensión de la actividad profesional porque pertenecen a grupos de infección de alto riesgo. Se desconocen aún las consecuencias que tiene el encierro en este grupo de profesionales. ${ }^{4,5}$

El objetivo de este trabajo fue describir la frecuencia de estrés, burnout, ansiedad y depresión en una muestra de médicos del Hospital de Clínicas José de San Martín, dependiente de la Facultad de Medicina de la Universidad de Buenos Aires durante la cuarentena por la pandemia de la COVID-19 y analizar las asociaciones con distintas variables independientes.

\section{MATERIALES Y MÉTODOS}

Se realizó un estudio observacional transversal en el mes de mayo de 2020. La muestra estuvo compuesta por médicos de un hospital universitario y polivalente.

Los criterios de inclusión fueron todos los médicos de planta, residentes y médicos pertenecientes a posiciones de liderazgo de las áreas clínicas, quirúrgicas, áreas cerradas, y de diagnóstico, pediatría y neonatología que firmaron el consentimiento informado.

Como criterio de exclusión se consideró la ausencia de los médicos en el momento del estudio y aquellos que no respondieron a los cuestionarios en forma total o parcial.

La encuesta se realizó en Buenos Aires, Argentina, cuando la situación no era tan crítica y no se había llegado al pico de casos. El hospital donde se tomó la muestra tenía, al momento de la realización de este estudio, cuatro unidades de internación reservadas para pacientes con COVID-19 y había recibido un total de 314 pacientes infectados.

Se realizó un cuestionario compuesto por dos secciones. La primera sección consistió en un temario sociodemográfico en el que se consignaron las siguientes variables independientes: edad, estado civil, puesto de trabajo, actividades realizadas en el hospital durante la pandemia, años de antigüedad, especialidad médica, trabajo exclusivo en la unidad de urgencias, trabajo en salas de pacientes con diagnóstico de COVID-19, si se encontraban adecuadamente entrenados para estar en contacto con pacientes con probable infección por COVID-19, si se encontraban exentos de atender pacientes por padecer factores de riesgo, existencia de lucro cesante, presencia de síntomas transitorios similares a la infección por COVID-19 y comienzo de ingesta de ansiolíticos o aumento de la dosis de estos.

La segunda sección constó de tres escalas: el Inventario de estrés de profesiones de la salud (HPSI, por su sigla en inglés), el Inventario de burnout de Maslach (MBI, por su sigla en inglés) y la Escala de ansiedad y depresión hospitalaria (HADS, por su sigla en inglés). Todas están validadas en español. ${ }^{6-8}$

El HPSI es un instrumento desarrollado para evaluar los niveles y fuentes de estrés que experimentan los profesionales de la salud. Es un cuestionario autoadministrado que consta de 30 situaciones laborales que los profesionales de la salud podrían encontrar en su práctica diaria. Los encuestados deben indicar con qué frecuencia encuentran cada situación estresante en su entorno laboral, utilizando una escala tipo Likert de 5 puntos (de nunca a muy a menudo). Cada elemento se puntúa de 0 a 4; el rango posible de puntuaciones en todo el HPSI es de 0 a $120 .{ }^{9}$

Para esta investigación se consideró un análisis dicotómico: la ausencia de estrés ( $\leq 30$ puntos) y la presencia de estrés ( $\geq 31$ puntos).

El MBI es un cuestionario psicológico introspectivo que consta de 22 ítems que evalúan el burnout. Mide tres dimensiones: agotamiento emocional, despersonalización y falta de realización personal. Se consideró presencia de burnout con una puntuación $\geq 26$ en agotamiento, $\geq 9$ en despersonalización y/o $\leq 36$ en logros personales. ${ }^{78}$

La HADS ${ }^{10}$ es una escala compuesta por 14 ítems. Está diseñada para la pesquisa rápida de síntomas de depresión (7 ítems) y ansiedad (7 ítems), entre poblaciones no psiquiátricas, médicamente enfermas, pacientes ambulatorios y la población general. ${ }^{11,12}$ Zigmond y Snaith establecieron que, en las subescalas de ansiedad y depresión, una puntuación de 0 a 7 es negativa, de 8 a 10 puntos es una caso posible y superior a 11 es un caso positivo. ${ }^{10,13}$ En este estudio se consideró una puntuación de corte $\geq 8$ para el cribado de ansiedad y depresión.

Todos los instrumentos mencionados se enviaron a través de Google Forms ${ }^{\circledR}$ a 440 médicos (de planta, personal jerárquico y residentes) del mismo hospital, del 6 al 11 de mayo de 2020 durante la pandemia de COVID-19, segundo mes de cuarentena en la República Argentina. El consentimiento informado se firmó electrónicamente antes de completar el cuestionario. 


\section{Análisis estadístico}

Se efectuó un análisis descriptivo de cada variable: sexo, edad, posición laboral, antigüedad, especialidad médica, concurrencia al hospital durante la pandemia, lucro cesante, atención de pacientes con sospecha o diagnóstico confirmado de COVID-19, percepción de no estar adecuadamente capacitado para estar en contacto con pacientes con infección probable de COVID-19, estar exceptuado de atender pacientes por presentar factores de riesgo médicos, presencia de síntomas transitorios similares a infección por COVID-19, comienzo de la toma de ansiolíticos o incremento de la dosis y realización de guardias de 24 horas en búsqueda de asociaciones bivariadas. Se utilizaron pruebas no paramétricas (chi cuadrado y prueba exacta de Fisher) para las variables cuantitativas en busca de asociación. Se consideró estadísticamente significativo un valor de $p<0,05$. Se utilizó el programa SPSS ${ }^{\circledR}$ para Mac ${ }^{\circledR}$, versión 24.

\section{Consideraciones éticas}

La investigación se realizó en cumplimiento de la Declaración de Helsinki y de la ley de habeas data, y fue aprobada por el Comité de Ética de Investigación Clínica del Hospital de Clínicas José de San Martín.

\section{RESULTADOS}

El flujograma (Figura 1) muestra el proceso de selección y evaluación de los participantes.

Los datos sociodemográficos se muestran en la Tabla 1. Los resultados de la prevalencia de estrés (HPSI), burnout (MBI) y ansiedad y depresión (HADS) se pueden encontrar en la Tabla 2.

\section{Análisis de escala HPSI}

Sobre un total de 440 médicos encuestados, $305(69,38 \%)$ respondieron el cuestionario de estrés de Wolfgang.

La prevalencia total de estrés hallada fue del 93,7 \% (IC95\%: 90,33-96,2).

Una puntuación positiva para el estrés se asoció significativamente con la asistencia al hospital con regularidad durante la pandemia, tener síntomas transitorios similares a COVID-19 e iniciar la toma de ansiolíticos o aumentar la dosis (Tabla 2).

\section{Análisis de la escala MBI}

Sobre un total de 440 médicos encuestados, $302(68,63 \%)$ respondieron el cuestionario de Maslach.

La prevalencia del burnout fue del 73,5\% (IC95 \%: 68,2-78,4); los médicos que obtuvieron un puntaje positivo eran significativamente más

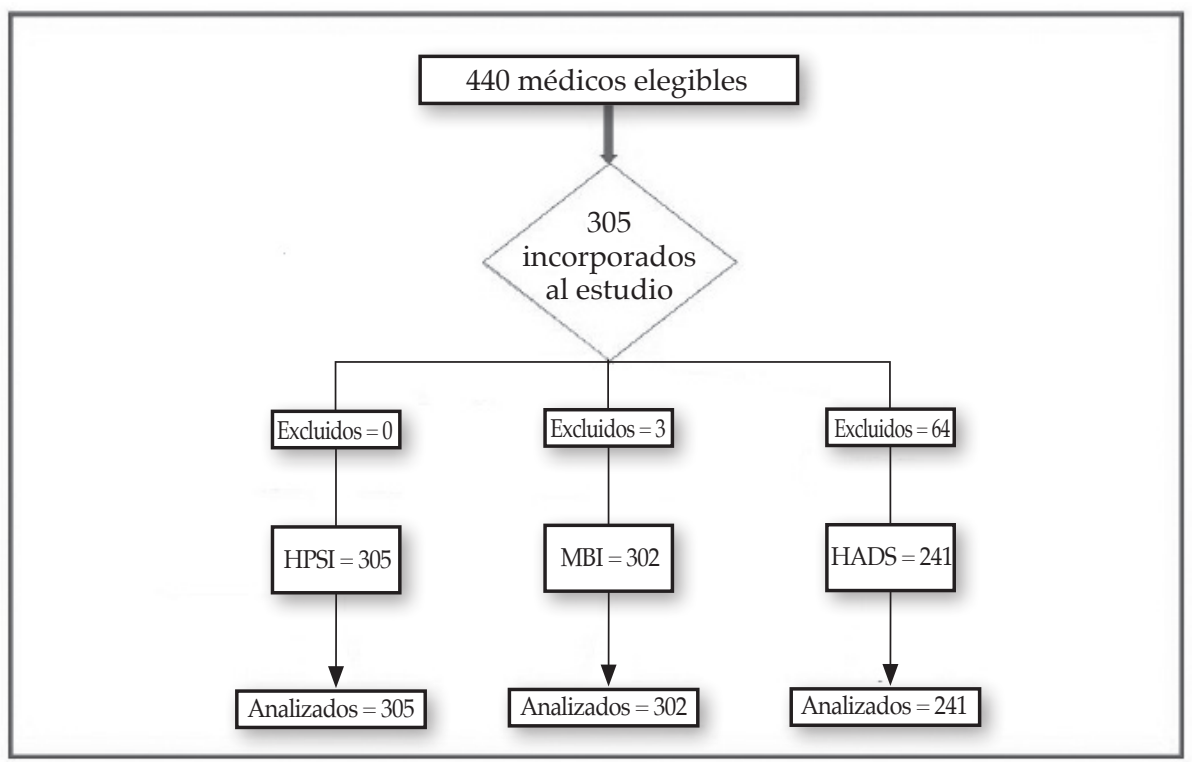

HPSI: Inventario de estrés de profesiones de la salud (por su sigla en inglés), MBI: Inventario de burnout de Maslach (por su sigla en inglés), HADS: Escala de ansiedad y depresión hospitalaria (por su sigla en inglés). 
jóvenes que los que no puntuaban para esta patología.

La presencia de síndrome de desgaste profesional se asoció con menor antigüedad, ser médico residente, atender pacientes con infección probable o definitiva por COVID-19, tener síntomas transitorios similares a COVID-19 y trabajar en turnos de 24 horas (Tabla 2).

\section{Análisis de la escala HADS}

Sobre un total de 440 médicos encuestados, $241(55 \%)$ respondieron la escala HADS.

La prevalencia de ansiedad fue del $44 \%$ (IC 95\%: 17,3-26,9) y la prevalencia de la depresión fue del 21,9\% (IC95 \%: 38,4-49,8). Todos los médicos que obtuvieron una puntuación alta en ansiedad también obtuvieron una puntuación alta en depresión.

La presencia de síntomas de ansiedad y depresión tuvo una asociación negativa con la antigüedad laboral. Dicho de otro modo, a menor antigüedad laboral, se halló mayor presencia de síntomas de ansiedad y depresión. De hecho, los médicos residentes tuvieron una frecuencia aún mayor de síntomas de ansiedad y depresión que el resto de los médicos evaluados.

La situación de atender a pacientes sospechosos o infectados con SARS-CoV-2 se relacionó con los síntomas de ansiedad únicamente.

TABLA 1. Datos sociodemográficos obtenidos durante la pandemia de COVID-19

\begin{tabular}{|c|c|c|}
\hline \multicolumn{3}{|l|}{$\begin{array}{l}\text { Demografía } \\
n=302\end{array}$} \\
\hline Sexo & $\begin{array}{l}\text { Masculino } \\
\text { Femenino }\end{array}$ & $\begin{array}{l}147(51,3 \%) \\
155(48,7 \%)\end{array}$ \\
\hline Edad $( \pm \mathrm{DE})$ & $\begin{array}{l}\text { Masculino } \\
\text { Femenino }\end{array}$ & $\begin{array}{l}44.42 \pm 11,58 \\
42.07 \pm 12,42\end{array}$ \\
\hline Posición laboral & $\begin{array}{l}\text { Posiciones de liderazgo } \\
\text { Médicos de planta } \\
\text { Médicos residentes }\end{array}$ & $\begin{array}{l}47(15,6 \%) \\
152(50,3 \%) \\
103(34,1 \%)\end{array}$ \\
\hline Antigüedad & $\begin{array}{l}0-5 \text { años } \\
6-15 \text { años } \\
>16 \text { años }\end{array}$ & $\begin{array}{l}97(32,1 \%) \\
69(22,8 \%) \\
136(45 \%)\end{array}$ \\
\hline Tipo de especialidad médica & $\begin{array}{l}\text { Clínica } \\
\text { Quirúrgica } \\
\text { Solo trabajo en emergencias } \\
\text { Sin contacto directo con los pacientes }\end{array}$ & $\begin{array}{c}163(54 \%) \\
117(38,7 \%) \\
10(3,3 \%) \\
12(4,1 \%)\end{array}$ \\
\hline Concurrencia al hospital durante la pandemia & $\begin{array}{l}\text { Sí } \\
\text { No }\end{array}$ & $\begin{array}{c}245(81 \%) \\
56(19 \%)\end{array}$ \\
\hline Lucro cesante & $\begin{array}{l}\text { Sí } \\
\text { No }\end{array}$ & $\begin{array}{l}152(51 \%) \\
146(49 \%)\end{array}$ \\
\hline $\begin{array}{l}\text { Atención de pacientes con sospecha o } \\
\text { diagnóstico confirmado de COVID-19 }\end{array}$ & $\begin{array}{l}\text { Sí } \\
\text { No }\end{array}$ & $\begin{array}{l}138(45,7 \%) \\
164(54,3 \%)\end{array}$ \\
\hline $\begin{array}{l}\text { Percepción de no estar adecuadamente capacita } \\
\text { para estar en contacto con pacientes con } \\
\text { infección probable por SARS-CoV-2 }\end{array}$ & $\begin{array}{l}\text { Sí } \\
\text { No }\end{array}$ & $\begin{array}{l}196(64,9 \%) \\
106(35,1 \%)\end{array}$ \\
\hline $\begin{array}{l}\text { Exceptuado de atender pacientes por } \\
\text { presentar factores de riesgo médicos }\end{array}$ & $\begin{array}{l}\text { Sí } \\
\text { No }\end{array}$ & $\begin{array}{l}44(15,23 \%) \\
256(84,7 \%)\end{array}$ \\
\hline $\begin{array}{l}\text { Presencia de síntomas transitorios similares } \\
\text { a infección por SARS-CoV-2 }\end{array}$ & $\begin{array}{l}\text { Sí } \\
\text { No }\end{array}$ & $\begin{array}{l}113(37,4 \%) \\
189(62,6 \%)\end{array}$ \\
\hline $\begin{array}{l}\text { Comienzo de la toma de ansiolíticos o } \\
\text { incremento de la dosis }\end{array}$ & $\begin{array}{l}\text { Sí } \\
\text { No }\end{array}$ & $\begin{array}{c}43(14,2 \%) \\
259(85,8 \%)\end{array}$ \\
\hline Guardias de 24 horas & $\begin{array}{l}\text { Sí } \\
\text { No }\end{array}$ & $\begin{array}{l}133(44 \%) \\
169(56 \%)\end{array}$ \\
\hline
\end{tabular}


Los síntomas de ansiedad y depresión también se asociaron significativamente con síntomas transitorios que se asemejan a la infección por SARS-CoV-2, a la toma de ansiolíticos y a trabajar en guardias de emergencia con turnos de 24 horas (Tabla 2).

\section{DISCUSIÓN}

La alta prevalencia de estrés, agotamiento, ansiedad y síntomas depresivos encontrados en este estudio -dadas las condiciones habituales de trabajo de los médicos en hospitales- no es inesperada. Además, la pandemia se ha relacionado con diversos factores sobreagregados que pueden contribuir a estos hallazgos, tales como la gran carga de trabajo, los dilemas morales y un ambiente laboral totalmente diferente al de la prepandemia. ${ }^{14}$

Los médicos suelen trabajar con altos niveles de estrés que los hacen propensos al burnout, a la ansiedad y a la depresión. Por lo tanto, se espera que, dadas las condiciones en las que se desarrolla la pandemia, los médicos se encuentren bajo una mayor presión y mayor riesgo de incrementar los niveles de estos cuadros.

En un análisis previo realizado en la misma

TABla 2. Análisis de las variables independientes en relación con el estrés, el burnout, la ansiedad y la depresión

\begin{tabular}{|c|c|c|c|c|c|c|c|c|c|}
\hline \multirow{2}{*}{\multicolumn{2}{|c|}{$\begin{array}{l}\text { Variables } \\
\text { independientes }\end{array}$}} & \multicolumn{2}{|c|}{ HPSI } & \multicolumn{2}{|c|}{ MBI } & \multicolumn{2}{|c|}{ HADS Ansiedad } & \multicolumn{2}{|c|}{ HADS Depresión } \\
\hline & & \multirow{2}{*}{$\begin{array}{l}\text { Estrés } \\
148(95,5 \%)\end{array}$} & \multirow{3}{*}{$\begin{array}{l}\begin{array}{l}\text { Resultados } \\
\text { estadisticos }\end{array} \\
x^{2}: 1,702 \\
p: 0,238\end{array}$} & \multirow{2}{*}{$\begin{array}{l}\text { Burnout } \\
107(72,8 \%)\end{array}$} & $\begin{array}{l}\text { Resultados } \\
\text { estadisticos }\end{array}$ & \multirow{2}{*}{$\begin{array}{l}\text { Ansiedad } \\
27(18,4 \%)\end{array}$} & \begin{tabular}{|l|} 
Resultados \\
estadisticos
\end{tabular} & \multirow{2}{*}{$\begin{array}{l}\text { Depresión } \\
38(44 \%)\end{array}$} & $\begin{array}{l}\text { Resultados } \\
\text { estadisticos }\end{array}$ \\
\hline \multirow[t]{2}{*}{ Sexo } & Masculino & & & & \multirow{2}{*}{$\begin{array}{l}x^{2}: 0,76 \\
p: 0,796\end{array}$} & & \multirow{2}{*}{$\begin{array}{l}X^{2}: 2,039 \\
p: 0,198\end{array}$} & & \multirow{2}{*}{$\begin{array}{l}X^{2}: 3,221 \\
p: 0,93\end{array}$} \\
\hline & Femenino & $\begin{array}{l}138 \\
(91,8 \%)\end{array}$ & & $\begin{array}{l}115 \\
(74,2 \%)\end{array}$ & & $\begin{array}{l}39 \\
(25,2 \%)\end{array}$ & & $76(49 \%)$ & \\
\hline \multirow[t]{3}{*}{ Antigūedad (años) } & $0-5$ & $92(94,8 \%)$ & \multirow[t]{3}{*}{$x^{2}: 0,501$} & $86(88,7 \%)$ & \multirow[t]{3}{*}{$x^{2}: 26,389$} & $32(33 \%)$ & \multirow[t]{3}{*}{$x^{2}: 11,993$} & $58(58,9 \%)$ & \multirow[t]{2}{*}{$x^{2}: 17,328$} \\
\hline & 6-15 & $65(94,2 \%)$ & & $55(79,5 \%)$ & & $\begin{array}{l}15 \\
(21,7 \%)\end{array}$ & & $31(44,9 \%)$ & \\
\hline & $\geq 16$ & $\begin{array}{l}126 \\
(92,6 \%)\end{array}$ & & $81(59,6 \%)$ & & $19(14 \%)$ & & $44(32,4 \%)$ & $p<0,001^{*}$ \\
\hline \multirow[t]{3}{*}{ Posición laboral } & Liderazgo & $42(89,4 \%)$ & \multirow{3}{*}{$\begin{array}{l}X^{2}: 3,731 \\
p: 0,198\end{array}$} & $\begin{array}{l}100 \\
(65,8 \%)\end{array}$ & \multirow[t]{3}{*}{$x^{2}: 22,914$} & $6(12,8 \%)$ & \multirow[t]{3}{*}{$x^{2}: 10,019$} & $10(21,3 \%)$ & \multirow[t]{3}{*}{$x^{2}: 23,425$} \\
\hline & Planta & $\begin{array}{l}141 \\
(92,6 \%)\end{array}$ & & $29(61,7 \%)$ & & $\begin{array}{l}22 \\
(17,8 \%)\end{array}$ & & $60(39,5 \%)$ & \\
\hline & Residentes & $\begin{array}{l}100 \\
(97,1 \%)\end{array}$ & & $93(90,3 \%)$ & & $33(32 \%)$ & & $63(61,2 \%)$ & \\
\hline \multirow[t]{4}{*}{ Especialidad médica } & Clínica & $\begin{array}{l}152 \\
(93,3 \%)\end{array}$ & \multirow{4}{*}{$\begin{array}{l}X^{2}: 0,833 \\
p: 0,778\end{array}$} & $\begin{array}{l}121 \\
(74,2 \%)\end{array}$ & $\begin{array}{l}\text { Test exacto } \\
\text { de Fisher }\end{array}$ & $\begin{array}{l}39 \\
(23,9 \%)\end{array}$ & $\begin{array}{l}\text { Test exacto } \\
\text { de Fisher }\end{array}$ & $75(45,4 \%)$ & $\begin{array}{l}\text { Test } \\
\text { exacto de }\end{array}$ \\
\hline & Quirúrgica & $110(94 \%)$ & & $86(73,5 \%)$ & $p: 0,644$ & $\begin{array}{l}23 \\
(19,7 \%)\end{array}$ & p: 0,813 & $50(42,7 \%)$ & $\begin{array}{l}\text { Fisher } \\
p: 0,391\end{array}$ \\
\hline & Emergencias & $10(100 \%)$ & & $8(80 \%)$ & & $2(20 \%)$ & & $6(60 \%)$ & \\
\hline & Sin contacto & $11(91,7 \%)$ & & $7(58,3 \%)$ & & $2(16,7 \%)$ & & $3(25 \%)$ & \\
\hline Concurre al hospital^ & Si & $\begin{array}{l}234 \\
(95,5 \%)\end{array}$ & $x^{2}: 5,202$ & $\begin{array}{l}185 \\
(75,5 \%)\end{array}$ & $x^{2}: 2,943$ & $\begin{array}{l}10 \\
(17,9 \%)\end{array}$ & $x^{2}: 0,666$ & $21(37,5 \%)$ & $x^{2}: 1,247$ \\
\hline & No & $49(87,5 \%)$ & $p: 0,049^{*}$ & $36(64,3 \%)$ & p: 0,095 & $\begin{array}{l}56 \\
(22,9 \%)\end{array}$ & $p: 0,478$ & $\begin{array}{l}112 \\
(45,7 \%)\end{array}$ & $p: 0,167$ \\
\hline Lucro cesante & Si & $\begin{array}{l}137 \\
(93,8 \%)\end{array}$ & $x^{2}: 0,21$ & $\begin{array}{l}106 \\
(69,7 \%)\end{array}$ & $x^{2}: 1,845$ & $\begin{array}{l}33 \\
(22,6 \%)\end{array}$ & $x^{2}: 0,215$ & $68(46,6 \%)$ & $x^{2}: 1,260$ \\
\hline & No & $142(93,4 \%)$ & p: 0,999 & $112(76,7 \%)$ & $p: 0,192$ & $31(20,4 \%)$ & $p: 0,674$ & $61(40,1 \%)$ & $p: 0,293$ \\
\hline Asiste a pacientes COVID. & Si & $154(93,9 \%)$ & $x^{2}: 0,23$ & $109(79,6 \%)$ & $x^{2}: 4,858$ & $39(28,5 \%)$ & $x^{2}: 6,283$ & $65(39,6 \%)$ & $x^{2}: 3,027$ \\
\hline $19 \mathrm{~m}$ & No & $129(93,5 \%)$ & p: 0,99 & $112(68,3 \%)$ & $p: 0,036^{\circ}$ & $27(16,5 \%)$ & $p: 0,017^{*}$ & $68(49,6 \%)$ & p: 0,103 \\
\hline Autopercepción de & Sí & $103(97,2 \%)$ & $x^{2}: 3,31$ & $135(68,9 \%)$ & $x^{2}: 6,154$ & $26(24,5 \%)$ & $x^{2}: 0,684$ & $52(49,1 \%)$ & $x^{2}: 1,668$ \\
\hline entrenamiento adecuado & No & $180(91,2 \%)$ & p: 0,08 & $87(82,1 \%)$ & $p: 0,014^{*}$ & $40(20,4 \%)$ & $p: 0,466$ & $81(41,3 \%)$ & $p: 0.225$ \\
\hline Exceptuado por factores & Si & $44(15,23 \%)$ & $x^{2}: 0,532$ & $31(67,4 \%)$ & $x^{2}: 1,043$ & $59(23 \%)$ & $x^{2}: 1,400$ & $114(44,5 \%)$ & $x^{2}: 0,165$ \\
\hline de riesgo & No & $256(84,7 \%)$ & $p: 0,51$ & $191(74,6 \%)$ & $p: 0,364$ & $7(15,2 \%)$ & $p: 0,255$ & $19(41,3 \%)$ & $p: 0,748$ \\
\hline Sintomas transitorios & Si & $111(98,2 \%)$ & Test exacto & $96(85 \%)$ & $x^{2}: 12,148$ & $34(30,1 \%)$ & $x^{2}: 7,168$ & $60(53,1 \%)$ & $x^{2} ; 6,011$ \\
\hline similares a COVID-19 & No & $172(91 \%)$ & $\begin{array}{l}\text { de Fisher } \\
\text { Test, p: } 0,01 \text { * }\end{array}$ & $126(66,7 \%)$ & $p: 0,001^{*}$ & $32(16,9 \%)$ & $p: 0,009^{*}$ & $73(38,6 \%)$ & $p: 0,017^{*}$ \\
\hline Ansioliticost & Si & $43(100 \%)$ & & $37(86 \%)$ & $X^{2}: 4,047$ & $21(48,8 \%)$ & $x^{2}: 21,375$ & $32(74,4 \%)$ & $X^{2}: 18,776$ \\
\hline & No & $240(92,7 \%)$ & & $185(71,4 \%)$ & $p: 0,06$ & $45(17,4 \%)$ & $p<0,001$ & $101(39 \%)$ & $p<0,001^{*}$ \\
\hline Trabajo en guardias de & Sí & $129(97 \%)$ & Test exacto & $119(89,5 \%)$ & $x^{2}: 31,104$ & $40(30,1 \%)$ & $x^{2}: 9,405$ & $78(58,6 \%)$ & $x^{2}: 20,576$ \\
\hline 24 horas & No & $154(91,1 \%)$ & $\begin{array}{l}\text { de Fisher } \\
p: 0.054\end{array}$ & $103(60,9 \%)$ & $P<0,001^{*}$ & $26(15,4 \%)$ & p: $0,003^{*}$ & $55(32,5 \%)$ & $p<0,001^{*}$ \\
\hline
\end{tabular}

HPSI: Inventario de estrés de profesiones de la salud (por su sigla en inglés), MBI: Inventario de burnout de Maslach (por su sigla en inglés), HADS: Escala de ansiedad y depresión hospitalaria (por su sigla en inglés), X²: prueba de chi cuadrado.

${ }^{*} p<0,05 ; \wedge$ acudir al hospital durante las pandemias; ${ }^{\wedge}$ : pacientes sospechosos o infectados por COVID-19;

\# inicio o aumento de dosis. 
institución durante noviembre de 2019, se halló una prevalencia muy alta de estrés y burnout (86\% y $73 \%$, respectivamente); además, un $10 \%$ de los participantes puntuó positivo en psicopatología en el GHQ-12. ${ }^{15}$ La prevalencia de estrés aumentó en el Hospital de Clínicas José de San Martín durante la pandemia.

Por otra parte, una revisión sistemática realizada por Rothenstein et al., en 2018 informó una prevalencia del $67 \%$ del síndrome de desgaste profesional entre los médicos. Esto demuestra que la población médica tiene, en condiciones basales, una prevalencia elevada de estrés, de síndrome de desgaste profesional y de ansiedad que, ante una situación de pandemia o desastre, la volvería particularmente vulnerable. ${ }^{14,16}$

El presente estudio halló que los médicos residentes están más expuestos al burnout, la ansiedad y la depresión en comparación con los médicos de planta y con aquellos que se desempeñan en posiciones de liderazgo. Es bien sabido que los residentes tienen jornadas laborales extensas con demandas muy altas y, a menudo, están sujetos a una sobrecarga de trabajo y de presión. ${ }^{17}$

Un trabajo realizado en Saint Louis (Estados Unidos) observó -de manera similar a lo hallado en este estudio- que los médicos residentes que estuvieron expuestos a la atención de pacientes con COVID-19 tenían una mayor prevalencia de depresión, ansiedad y estrés que la población general; además, mostraron una mayor tasa de síndrome de desgaste profesional que el grupo no expuesto. ${ }^{18}$

Los altos niveles de estrés y agotamiento se han asociado con un mayor riesgo de trastornos psicopatológicos, especialmente trastornos afectivos y de ansiedad. Entre los trastornos de ansiedad, el trastorno de estrés postraumático puede ser una de las consecuencias más temidas del estrés y del síndrome de desgaste profesional, porque tiende a la cronicidad cuando no se trata de forma prematura, con un gran impacto negativo en quienes lo padecen. ${ }^{19,20}$

Otro hallazgo de este estudio fue que quienes trabajan en sectores de emergencia con guardias de 24 horas tienen altos niveles de estrés y agotamiento. Es importante resaltar que, en el Hospital de Clínicas José de San Martín, luego del segundo año de formación, los residentes también realizan turnos de 24 horas en el Departamento de Emergencias. Según estos hallazgos, el grupo de jóvenes residentes que trabajan en la sala de emergencias puede ser vulnerable a los impactos en la salud mental durante la pandemia por COVID-19. Desde el punto de vista preventivo, es importante poner una especial atención en los residentes y en los médicos que realizan turnos de emergencia de 24 horas.

Los cuestionarios sociodemográficos proporcionados mostraron, además, una alta prevalencia de síntomas transitorios compatibles con la infección por COVID-19. Este hallazgo se correlacionó con una prevalencia significativa de estrés, agotamiento, ansiedad y depresión. Lo mismo ocurrió con los médicos que iniciaron la ingesta de ansiolíticos o aumentaron la dosis. Ambos hallazgos pueden tener la ansiedad como un factor común. Se sabe que los síntomas por somatización se vinculan a incrementos de estrés, síndrome de desgaste profesional, ansiedad y depresión. Cabe agregar que durante la pandemia por COVID-19 existe un aumento en la percepción de situaciones de amenaza e incertidumbre en el personal de salud. Estos son factores fundamentales en el proceso de generación de síntomas por somatización, ${ }^{21,22}$ como, por ejemplo, la percepción de síntomas transitorios de COVID-19.

Sin embargo, no es posible concluir que los síntomas transitorios se debieran a síntomas de somatización. De todos modos, esta especulación sobre la vinculación del estrés y las reacciones de somatización durante la pandemia configura un tema que requiere mayor investigación.

Los cuadros evaluados en este estudio también han sido investigados en otros centros. Por ejemplo, en un estudio realizado en Milán, el $71,2 \%$ de los médicos encuestados presentaba rasgos de ansiedad según el Cuestionario de ansiedad estado-rasgo; el 26,8 \% tenía depresión clínica, el 31,3 \% tenía ansiedad clínica; el 34,3 \% tenía estrés y más del $30 \%$, síndrome de desgaste profesional. Estos porcentajes se asociaron con largas jornadas laborales, la presencia de comorbilidades psicológicas, el miedo a contagiarse de COVID-19 y la percepción de la falta de apoyo por parte de los amigos. ${ }^{23}$

Otra investigación realizada en España durante la pandemia por COVID-19 mostró un alto porcentaje de profesionales que estaban emocionalmente agotados. En la escala HADS, utilizando una puntuación de corte de 8 , el $58,6 \%$ de los profesionales tenía síntomas de ansiedad y el $46 \%$, síntomas depresivos. Los turnos nocturnos o los turnos de 12 y 24 horas se vincularon con el trastorno de 
estrés postraumático y la depresión; el hecho de trabajar muchas horas a la semana se asoció con depresión; los trabajadores con menor capacitación tenían una mayor prevalencia de trastornos por estrés postraumático. Asimismo, las trabajadoras menos capacitadas tenían una mayor prevalencia de ansiedad, depresión y de trastorno por estrés postraumático. ${ }^{24}$

En un estudio multicéntrico realizado en China, el 55,1\% de los profesionales tenía puntuaciones positivas para el estrés $\mathrm{y}$, utilizando una puntuación de corte de 8 en la escala HADS, el $54,2 \%$ padecía síntomas de ansiedad y el $58 \%$, síntomas de depresión. Los médicos subalternos e intermedios y los que tenían menos de 11 años de antigüedad -al igual que en la presente investigación- se asociaron con niveles de estrés elevados. Por otra parte, los síntomas de ansiedad y depresión se relacionaron con tres factores: no contar con equipo de protección adecuado, estar en contacto con pacientes diagnosticados o sospechosos de COVID-19 y ser mujer. ${ }^{25}$

Por último, un metaanálisis realizado por investigadores griegos mostró que la prevalencia de ansiedad en los profesionales de la salud durante la pandemia por COVID-19 fue del $23,32 \%$; la prevalencia de depresión fue del $22,8 \%$ y el $34,32 \%$ presentaba insomnio. ${ }^{26}$

Una de las limitaciones de este estudio es que los resultados muestran la situación dos meses después del comienzo de la cuarentena. Se obtendría una imagen más precisa con un seguimiento regular durante la pandemia e incluso después de que esta haya finalizado.

Aunque se han publicado muchos artículos sobre esta situación, todavía no existe una perspectiva clara de las consecuencias en la salud mental de los trabajadores de la salud durante la pandemia. ${ }^{5,27}$

\section{CONCLUSIÓN}

En la muestra analizada, los residentes y quienes trabajan en emergencias en turnos de 24 horas mostraron porcentajes significativamente más altos de síndrome de desgaste profesional, ansiedad y depresión, en comparación con médicos de planta y con aquellos en posiciones de liderazgo. Estos hallazgos pueden estar asociados con una mayor carga de trabajo y una menor experiencia. Es mandatorio tomar medidas preventivas y terapéuticas para preservar a quienes hacen frente a esta pandemia.

\section{Agradecimientos}

A Marcelo Mazza por el soporte técnico, a Alejandra Schvartz y a Marcela Broner por la revisión crítica, y al profesor Patricio Jacovella por el apoyo para el desarrollo de este trabajo.

\section{REFERENCIAS}

1. Lipsitch M, Swerdlow DL, Finelli L. Defining the epidemiology of Covid-19-studies needed. N Engl J Med. 2020; 382(13):1194-6.

2. LancetT.COVID-19: protecting health-care workers. Lancet. 2020; 395(10228):922.

3. Spinelli A, Pellino G. COVID-19 pandemic: perspectives on an unfolding crisis. Br J Surg. 2020; 107(7):785-7.

4. Ferneini EM. The financial impact of COVID-19 on our practice. J Oral Maxillofac Surg. 2020; 78(7):1047-8.

5. Pfefferbaum B, North CS. Mental health and the Covid-19 pandemic. N Engl J Med. 2020; 383(6):510-2.

6. Palacios Nava M, Morán Álvarez I, Paz Román M. Validación del Inventario de Wolfgang en médicos mexicanos. REMESAT. 2014; 6(16):62-8.

7. Maslach C, Jackson SE, Leiter MP. Maslach Burnout Inventory Manual. 3rd ed. Palo Alto, CA: Consulting Psychologists Press; 1996.

8. Gil-Monte PR. Factorial validity of the Maslach Burnout Inventory (MBI-HSS) among Spanish professionals. Rev Sáude Publica. 2005; 39(1):1-8.

9. Wolfgang AP. The health professions stress inventory. Psychol Rep. 1988; 62(1):220-2.

10. Zigmond AS, Snaith RP. The hospital anxiety and depression scale. Acta Psychiatr Scand. 1983; 67(6):361-70.

11. Snaith RP. The hospital anxiety and depression scale. Health Qual Life Outcomes. 2003; 1:29.

12. Bjelland I, Dahl AA, Haug TT, Neckelmann D. The validity of the hospital anxiety and depression scale-an an updated literature review. J Psychosom Res. 2002; 52(2):69-77.

13. Gercovich N, Gercovich D, Torrente F, López P, et al. Adaptation and validation of the Hospital Anxiety and Depression Scale (HADS) to a cohort of patients in Buenos Aires, Argentina. J Clin Oncol. 2008; 26(15 Suppl):20616.

14. Shanafelt T, Ripp J, Trockel M. Understanding and Addressing Sources of Anxiety Among Health Care Professionals During the COVID-19 Pandemic. JAMA. 2020; 323(21):2133-4.

15. Duarte JM, Basile ME, Rodríguez Cairoli F, Appiani FJ. Prevalence of Stress, Burnout Syndrome and Psychopathology in Staff Physicians and those with Hierarchical Positions in a University Hospital. EC Neurology. 2020; 12(10):16-25.

16. Rotenstein LS, Torre M, Ramos MA, Rosales RC, et al. Prevalence of burnout among physicians: a systematic review. JAMA. 2018; 320(11):1131-50.

17. Dewa CS, Loong D, Bonato S, Trojanowski L, Rea M. The relationship between resident burnout and safety-related and acceptability-related quality of healthcare: a systematic literature review. BMC Med Educ. 2017; 17(1):195.

18. Kannampallil T, Goss C, Evanoff BA, Strickland JR, et al. Exposure to COVID-19 patients increases physician trainee stress and burnout. PLoS One. 2020; 15(8):e237301.

19. Restauri N, Sheridan AD. Burnout and Posttraumatic Stress Disorder in the Coronavirus disease 2019 (COVID-19) Pandemic: Intersection, Impact, and Interventions. J Am Coll Radiol. 2020; 17(7):921-6. 
20. Meis LA, Noorbaloochi S, Hagel Campbell EM, Erbes CR, et al. Sticking it out in trauma-focused treatment for PTSD: It takes a village. J Consult Clin Psychol. 2019; 87(3):246-56.

21. Dimsdale JE, Creed F, Escobar J, Sharpe M, et al. Somatic symptom disorder: an important change in DSM. J Psychosom Res. 2013; 75(3):223-8.

22. Glise K, Ahlborg G Jr, Jonsdottir IH. Prevalence and course of somatic symptoms in patients with stress-related exhaustion: does sex or age matter. BMC Psychiatry. 2014; 14:118.

23. Giusti E, Pedroli E, D’Aniello G, Stramba Badiale C, et al. The psychological impact of the COVID-19 outbreak on health professionals: a cross-sectional study. Front Psychol. 2020; $11: 1684$

24. Luceño-Moreno L, Talavera-Velasco B, García-Albuerne Y, Martín-García J. Symptoms of posttraumatic stress, anxiety, levels of resilience and burnout in Spanish health personnel during COVID-19 pandemic. Int J Environ Res Public Health. 2020; 17(15):5514.

25. Xiao X, Zhu X, Fu S, Hu Y, et al. Psychological impact of healthcare workers in China during COVID-19 pneumonia epidemic: A multi-center cross-sectional survey investigation. J Affect Disord. 2020; 274:405-10.

26. Pappa S, Ntella V, Giannakas T, Giannakoulis VG, et al. Prevalence of depression, anxiety, and insomnia among healthcare workers during the COVID-19 pandemic: a systematic review and meta-analysis. Brain Behav Immun. 2020:88:901-7.

27. Galbraith N, Boyda D, McFeeters D, Hassan T. The mental health of doctors during the Covid-19 pandemic. BJ Psych Bull. 2020; 1-4.

\section{Artículos seleccionados}

Los siguientes resúmenes y comentarios de trabajos seleccionados se encuentran disponibles en la versión electrónica de este número.

J Neuromuscul Dis. 2020;7(4):453-8.

Historia natural de la atrofia espinal tipo 1 en una serie de pacientes argentinos (Aguerre $V$, et al. Natural history of type 1 spinal muscular atrophy in a series of Argentinian children)

Comentario: Dr. Javier Muntadas. Sección Patología Neuromuscular Pediátrica. Hospital Italiano de Buenos Aires.

Pediatr Radiol. 2021 Apr 1:1-11.

Estudios de imágenes pulmonares en la enfermedad por coronavirus 2019: serie de 140 niños

latinoamericanos (Ugas-Charcape CF, et al. Pulmonary imaging in coronavirus disease 2019 (COVID-19): a series of 140 Latin American children)

Comentario: Dr. Leopoldo Lonegro. División Radiodiagnóstico. Hospital General de Niños Pedro de Elizalde. Ciudad de Buenos Aires.

Pediatr Pulmonol. 2021 Jun;56(6):1681-6.

Hallazgos clínicos, funcionales y en la tomografía computada en una cohorte de pacientes con hiperplasia de células neuroendócrinas de la infancia (Balinotti J, et al. Clinical, Functional and computed tomography findings in a cohort of patients with neuroendocrine cell hyperplasia of infancy) Comentario: Dr. Santiago M. Vidaurreta. Hospital Universitario CEMIC. Ciudad de Buenos Aires.

JAMA. 2020;324(11):1105-7.

Políticas entre los pediatras de EE. UU. para no atender pacientes que retrasan o rechazan la vacunación (O'Leary ST, et al. Policies among US pediatricians for dismissing patients for delaying or refusing vaccination)

Comentario: Dr. Norberto Giglio. Epidemiología, Hospital de Niños Ricardo Gutiérrez. Ciudad de Buenos Aires.

N Engl J Med. 2020 Jul 30;383(5):426-439.

Vacunación contra el virus sincicial respiatorio durante el embarazo y sus efectos en los niños (Madhi SA, et al. Respiratory syncytial virus vaccination during pregnancy and effects in infants)

Comentario: Dra. Angela Gentile. Hospital de Niños Ricardo Gutierrez. Ciudad de Buenos Aires. 